\title{
Structure of Tsai-type icosahedral quasicrystals
}

\author{
H. Takakura \\ Division of Applied Physics, Faculty of Engineering, Hokkado University, Sapporo, Hokkaido 060-8628, Japan \\ takakura@eng.hokudai.ac.jp
}

The Al-Mn icosahedral quasicrystal discovered by Shechtman was a metastable phase [1]. Attempts at its structure solution immediately began after the discovery was declared. After the subsequent discovery of stable icosahedral quasicrystals, the study of their physical properties became more active in addition to the elucidation of their structures, but these quasicrystals were all formed in ternary systems [2]. Because chemical disorder of inherent in ternary icosahedral quasicrystals, it was difficult to achieve any structure solution which is comparable to what is obtained for ordinaryl crystal. Discovery of a stable binary icosahedral quasicrystal in a $\mathrm{Cd}-\mathrm{Yb}$ alloy opened a route to the structure solution to at least for this particular type of quasicrystal [3]. This quasicrystal, now known as Tsai-type icosahedral quasicrystal, forms the largest group among known quasicrsytals and their approximant crystals forming systems. Although I was not directly involved in the discovery of this quasicrystal, I was present at the scene as a member of Tsai's group and took the first X-ray transmission Laue photographs (Fig. 1), which were appeared in the paper announcing the discovery [3]. In the beginning, the quality of Cd-Yb quasicrystal was not very good, but soon good quality was obtained, and higherdimensional crystal structure analysis by means of single crystal X-ray diffraction became possible [4]. Here is the story of how we arrived at the structural solution of this particular icosahedral quasicrystal.
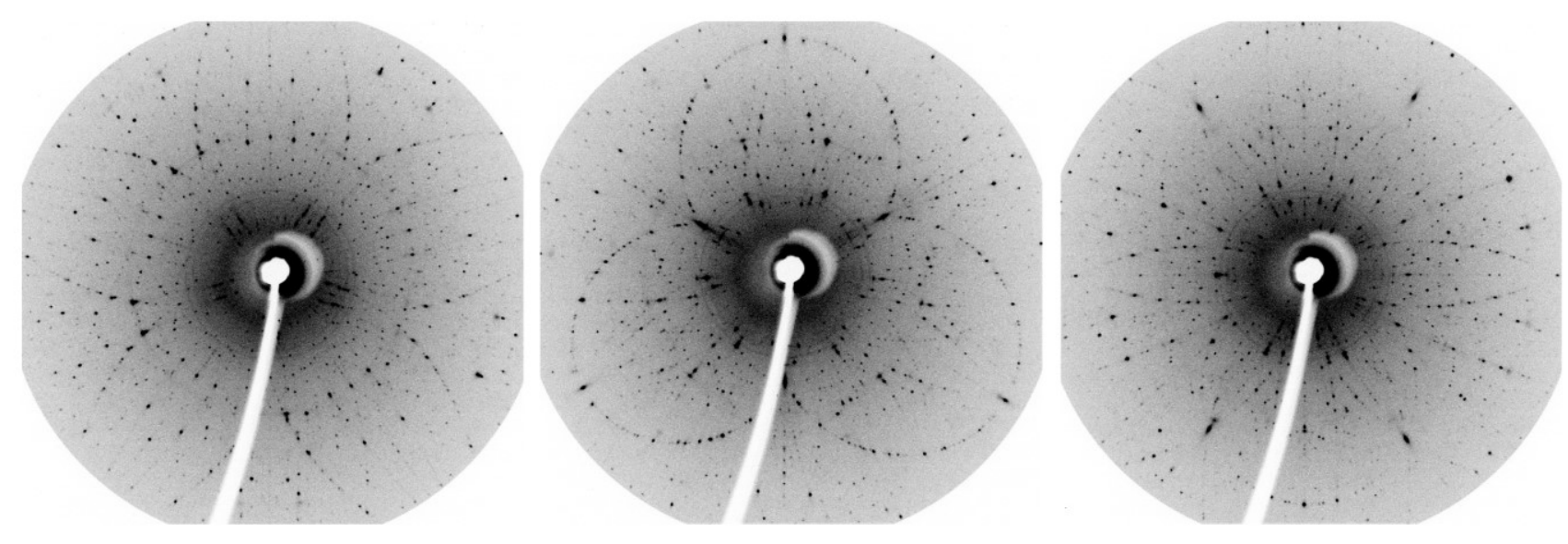

Figure 1. X-ray transmission Laue photographs of $\mathrm{Cd}-\mathrm{Yb}$ icosahedral quasicrystal along 5-fold, 3-fold and 2-fold-axes (from left to right).

[1] Shechtman, D., Blech, I., Gratias, D. \& Cahn, J. W. (1984). Phys. Rev. Lett. 53, 1951.

[2] Tsai, A. P., Inoue, A. \& Masumoto, T. (1989). Mater. Trans. JIM 30, 666.

[3] Tsai, A. P., Guo, J. Q., Abe, E., Takakura H. \& Sato, T. J. (2000). Nature 408, 537.

[4] Takakura, H., Gómez, C. P., Yamamoto, A., De Boissieu, M. \& Tsai, A. P. (2007). Nat. Mater. 6, 58.

\section{Keywords: Icosahedral quasicrystals; Tsai-type; Cd-Yb; structure solution}

The author is supported by JSPS KAKENHI Grant Numbers JP19H05817, JP19H05819, JP19K04982. 